\title{
Existence of Positive Solutions for Some Superlinear Fourth-Order Boundary Value Problems
}

\author{
Imed Bachar' ${ }^{1}$ and Habib Mâagli ${ }^{2}$ \\ ${ }^{1}$ Mathematics Department, College of Science, King Saud University, P.O. Box 2455, Riyadh 11451, Saudi Arabia \\ ${ }^{2}$ Department of Mathematics, College of Sciences and Arts, King Abdulaziz University, Rabigh Campus, P.O. Box 344, \\ Rabigh 21911, Saudi Arabia
}

Correspondence should be addressed to Imed Bachar; abachar@ksu.edu.sa

Received 30 May 2014; Accepted 12 July 2014; Published 11 August 2014

Academic Editor: Józef Banaś

Copyright (C) 2014 I. Bachar and H. Mâagli. This is an open access article distributed under the Creative Commons Attribution License, which permits unrestricted use, distribution, and reproduction in any medium, provided the original work is properly cited.

We are concerned with the following superlinear fourth-order equation $u^{(4)}(t)+u(t) \varphi(t, u(t))=0, t \in(0,1) ; u(0)=u(1)=$ $0, u^{\prime}(0)=a, u^{\prime}(1)=-b$, where $a, b$ are nonnegative constants such that $a+b>0$ and $\varphi(t, s)$ is a nonnegative continuous function that is required to satisfy some appropriate conditions related to a class $\mathscr{K}$ satisfying suitable integrability condition. Our purpose is to prove the existence, uniqueness, and global behavior of a classical positive solution to the above problem by using a method based on estimates on the Green function and perturbation arguments.

\section{Introduction}

A natural motivation for studying higher order BVPs lies in their applications. It is well-known (see for instance [1]) that the deformation of an elastic beam in equilibrium state, whose both ends clamped, can be described by fourth-order BVP

$$
u^{(4)}(t)=f(t, u(t)), \quad t \in(0,1),
$$

subject to the boundary conditions

$$
u(0)=u(1)=u^{\prime}(0)=u^{\prime}(1)=0 .
$$

When the nonlinearity $f$ is nonnegative such problems have been extensively investigated by many researchers, and various forms of the equation and boundary condition have been discussed; see, for example, [1-15] and references therein.

In particular, in 2005, Ma and Tisdel [1] studied the existence of positive solutions to the sublinear BVP

$$
\begin{aligned}
& u^{(4)}(t)=p(t) u^{\sigma}(t), \quad t \in(0,1), \\
& u(0)=u(1)=u^{\prime}(0)=u^{\prime}(1)=0,
\end{aligned}
$$

where $\sigma \in(0,1)$ and $p:(0,1) \rightarrow[0, \infty)$ is continuous which may be singular at both ends $t=0$ and $t=1$ and satisfying some integrable conditions. Using the method of lower and upper solutions for fourth-order boundary value problems, they have given some necessary and sufficient conditions for the existence of regular positive solutions to the boundary value problem (3).

In [8], the authors used fixed-point index results, to prove the existence of a positive solution for the boundary value problem

$$
\begin{gathered}
u^{(4)}(t)=p(t) f(u(t)), \quad t \in(0,1), \\
u(0)=u(1)=u^{\prime}(0)=u^{\prime}(1)=0,
\end{gathered}
$$

where $f:[0, \infty) \rightarrow[0, \infty)$ is nondecreasing continuous function allowed to be superlinear and $p:(0,1) \rightarrow[0, \infty)$ is continuous which may be singular at both ends $t=0$ and $t=1$ satisfying some adequate conditions.

Recently, in [4], the author considered problem (3), with $\sigma \in(-1,1)$ and $p$ is a nonnegative continuous function on $(0,1)$ satisfying some hypotheses related to Karamata regular variation theory. Using the Schauder fixed-point theorem, 
he established the existence and uniqueness of a positive solution to (3).

Here, by using a method based on estimates on the Green function and perturbation arguments, we show that, for negative nonlinearity $f$, problem (1) has a unique positive classical solution subject to some boundary conditions. More precisely, we are concerned with the following superlinear fourth order problem

$$
\begin{gathered}
u^{(4)}(t)+u(t) \varphi(t, u(t))=0, \quad t \in(0,1), \\
u(0)=u(1)=0, \quad u^{\prime}(0)=a, \quad u^{\prime}(1)=-b,
\end{gathered}
$$

where $a, b$ are nonnegative constants such that $a+b>0$. Our goal is to answer the questions of existence, uniqueness, and global behavior of a classical positive solution to problem (5), where the nonnegative nonlinear term $\varphi(t, s)$ is required to satisfy some appropriate conditions related to the following class $\mathscr{K}$.

Definition 1. A Borel measurable function $q$ in $(0,1)$ belongs to the class $\mathscr{K}$ if $q$ satisfies the following condition:

$$
\int_{0}^{1} r^{3}(1-r)^{3}|q(r)| d r<\infty
$$

We will often refer in this paper to

$$
\omega(t):=a t(1-t)^{2}+b t^{2}(1-t)
$$

the unique solution of the problem

$$
\begin{gathered}
u^{(4)}(t)=0, \quad t \in(0,1), \\
u(0)=u(1)=0, \quad u^{\prime}(0)=a, \quad u^{\prime}(1)=-b .
\end{gathered}
$$

Observe that, for $t \in[0,1]$,

$$
\min (a, b) \rho(t) \leq \omega(t) \leq \max (a, b) \rho(t)
$$

where $\rho(t):=t(1-t)$.

Also we denote by $G(t, s)$ the Green function of the operator $u \rightarrow u^{(4)}$, with the conditions $u(0)=u(1)=u^{\prime}(0)=$ $u^{\prime}(1)=0$, which can be explicitly given by

$$
\begin{aligned}
& G(t, s) \\
& \quad=\frac{1}{6} \begin{cases}t^{2}(1-s)^{2}[3 s-t(1+2 s)], & 0 \leq t \leq s \leq 1, \\
s^{2}(1-t)^{2}[3 t-s(1+2 t)], & 0 \leq s \leq t \leq 1,\end{cases} \\
& =\frac{1}{6}(t \wedge s)^{2}(1-t \vee s)^{2}[3(t \vee s)-(t \wedge s)(1+2(t \vee s))],
\end{aligned}
$$

where $t \wedge s=\min (t, s)$ and $t \vee s=\max (t, s)$ (see [2]).
The outline of the paper is as follows. In Section 2, we give some sharp estimates on Green's function $G(t, s)$, including the following 3G-inequality: for each $t, s, r \in(0,1)$,

$$
\begin{aligned}
& \frac{G(t, r) G(r, s)}{G(t, s)} \leq \frac{3}{2}\left[\left(\frac{\rho(r)}{\rho(t)}\right)^{2} G(t, r)+\left(\frac{\rho(r)}{\rho(s)}\right)^{2} G(r, s)\right], \\
&\left(\frac{\rho(r)}{\rho(t)}\right)^{2} G(t, r) \leq \frac{1}{2}(\rho(r))^{3},
\end{aligned}
$$

where $\rho(r)=r(1-r)$.

In particular, we derive from this $3 \mathrm{G}$-inequality that, for each $q \in \mathscr{K}$, one has

$$
\alpha_{q}:=\sup _{t, s \in(0,1)} \int_{0}^{1} \frac{G(t, r) G(r, s)}{G(t, s)}|q(r)| d r<\infty .
$$

In Section 3, our purpose is to study the superlinear fourth-order problem (5). The nonlinearity $\varphi$ is required to satisfy a combination of the following assumptions.

$\left(H_{1}\right) \varphi$ is a nonnegative continuous function in $(0,1) \times$ $[0, \infty)$.

$\left(H_{2}\right)$ There exists a nonnegative function $q \in \mathscr{K} \cap C(0,1)$ with $\alpha_{q} \leq 1 / 2$ such that for each $t \in(0,1)$, the map $s \rightarrow s(q(t)-\varphi(t, s \omega(t)))$ is nondecreasing on $[0,1]$.

$\left(H_{3}\right)$ For each $t \in(0,1)$, the function $s \rightarrow s \varphi(t, s)$ is nondecreasing on $[0, \infty)$.

We will first exploit the 3G-inequality to prove that the inverse of fourth-order operators that are perturbed by a zero-order term are positivity preserving. That is, if $\psi$ is a positive measurable function and $q$ is a nonnegative function belonging to the class $\mathscr{K} \cap(C(0,1))$ with $\alpha_{q} \leq 1 / 2$, then the following problem

$$
\begin{gathered}
u^{(4)}(t)+q(t) u(t)=\psi(t), \quad t \in(0,1), \\
u(0)=u(1)=u^{\prime}(0)=u^{\prime}(1)=0,
\end{gathered}
$$

has a positive solution. It turns out to prove that problem (13) admits a positive Green function $\mathscr{G}(t, s)$.

Based on the construction of this Green function and by using perturbation arguments, we prove the following.

Theorem 2. Assume $\left(H_{1}\right)-\left(H_{2}\right)$, then problem (5) has a positive solution $u$ in $C^{4}((0,1)) \cap C([0,1])$ satisfying

$$
c_{0} \omega(t) \leq u(t) \leq \omega(t), \quad t \in[0,1]
$$

where $c_{0}$ is a constant in $(0,1)$.

Moreover, if hypothesis $\left(\mathrm{H}_{3}\right)$ is also satisfied, then the solution $u$ to problem (5) satisfying (14) is unique.

Corollary 3. Let $f$ be a nonnegative function in $C^{1}([0, \infty))$ such that the map $s \rightarrow \theta(s)=s f(s)$ is nondecreasing on $[0, \infty)$. Let $p$ be a nonnegative continuous function on $(0,1)$ such that the function $t \rightarrow \widetilde{p}(t):=p(t) \max _{0 \leq \xi \leq \omega(t)} \theta^{\prime}(\xi)$ 
belongs to the class $\mathscr{K}$. Then for $\lambda \in\left[0,1 / 2 \alpha_{\tilde{p}}\right)$, the following problem

$$
\begin{gathered}
u^{(4)}(t)+\lambda p(t) u(t) f(u(t))=0, \quad t \in(0,1), \\
u(0)=u(1)=0, \quad u^{\prime}(0)=a, \quad u^{\prime}(1)=-b,
\end{gathered}
$$

has a unique positive solution $u$ in $C^{4}((0,1)) \cap C([0,1])$ satisfying

$$
\left(1-\lambda \alpha_{\tilde{p}}\right) \omega(t) \leq u(t) \leq \omega(t), \quad t \in[0,1]
$$

Observe that in Theorem 2, we obtain a positive classical solution $u$ to problem (5) whose behavior is not affected by the perturbed term. That is, it behaves like the solution $\omega$ of the homogeneous problem (8). As typical example of nonlinearity satisfying $\left(H_{1}\right)-\left(H_{3}\right)$, we quote $\varphi(t, s)=\lambda p(t) s^{\sigma}$, where $\sigma \geq 0, p$ is a positive continuous function on $(0,1)$ such that

$$
\int_{0}^{1} r^{3+\sigma}(1-r)^{3+\sigma} p(r) d r<\infty
$$

and $q(t)=\lambda \widetilde{p}(t):=\lambda(\sigma+1) p(t)(\omega(t))^{\sigma}$, with $\lambda \in\left[0,1 / 2 \alpha_{\tilde{p}}\right)$. Note that by using (9) and (17) the function $\widetilde{p}$ belongs to the class $\mathscr{K}$.

As usual, let $\mathscr{B}((0,1))$ be the set of Borel measurable functions in $(0,1)$ and let $\mathscr{B}^{+}((0,1))$ be the set of nonnegative ones.

We define the kernel $V: \mathscr{B}^{+}((0,1)) \rightarrow \mathscr{B}^{+}((0,1))$ by

$$
V \psi(t):=\int_{0}^{1} G(t, r) \psi(r) d r, \quad t \in[0,1] .
$$

Let $q \in \mathscr{B}^{+}((0,1))$. We define the kernel $V(q \cdot): \mathscr{B}^{+}$ $((0,1)) \rightarrow \mathscr{B}^{+}((0,1))$ by

$$
V(q)(\psi)(t):=\int_{0}^{1} G(t, r) q(r) \psi(r) d r, \quad t \in[0,1] .
$$

Remark 4 (See [1]). Let $\psi \in \mathscr{B}^{+}((0,1))$ such that the function $r \rightarrow(\rho(r))^{2} \psi(r)$ is continuous and integrable on $(0,1)$, then $V \psi$ is the unique solution in $C^{4}((0,1)) \cap C([0,1])$ of

$$
\begin{gathered}
u^{(4)}(t)=\psi(t), \quad t \in(0,1) ; \\
u(0)=u(1)=u^{\prime}(0)=u^{\prime}(1)=0 .
\end{gathered}
$$

\section{Estimates on the Green's Function}

From the explicit expression of Green's function (10) we derive the following.
Proposition 5 (see $[4,7])$. For $t, s \in[0,1]$, one has

$$
\begin{aligned}
\frac{1}{3}( & t \wedge s)^{2}(1-t \vee s)^{2}(t \vee s)(1-t \wedge s) \\
& \leq G(t, s) \\
& \leq \frac{1}{2}(t \wedge s)^{2}(1-t \vee s)^{2}(t \vee s)(1-t \wedge s),
\end{aligned}
$$

$$
\begin{aligned}
\frac{1}{3}(\rho(t))^{2}(\rho(s))^{2} & \leq G(t, s) \\
& \leq \frac{1}{2} \rho(t) \rho(s)(\rho(t) \wedge \rho(s))
\end{aligned}
$$

Using (22), we deduce the following.

Corollary 6. For $s, t \in(0,1)$, one has

(i)

$$
\left(\frac{\rho(s)}{\rho(t)}\right)^{2} G(t, s) \leq \frac{1}{2}(\rho(s))^{3}
$$

(ii) For $\psi \in \mathscr{B}^{+}((0,1))$, the function $t \rightarrow V \psi(t)$ is continuous on $[0,1]$ if and only if the integral $\int_{0}^{1}(\rho(s))^{2} \psi(s) d s$ converges.

Theorem 7 (3G inequality). For each $t, s, r \in(0,1)$, one has

$$
\frac{G(t, r) G(r, s)}{G(t, s)} \leq \frac{3}{2}\left[\left(\frac{\rho(r)}{\rho(t)}\right)^{2} G(t, r)+\left(\frac{\rho(r)}{\rho(s)}\right)^{2} G(r, s)\right],
$$

where $\rho(t)=t(1-t)$.

Proof. To prove the inequality, we denote by $A(t, s)=$ $(\rho(t) \rho(s))^{2} / G(t, s)$ and we claim that $A$ is a quasi-metric, that is, for each $t, s, r \in(0,1)$,

$$
A(t, s) \leq \frac{3}{2}[A(t, r)+A(r, s)] .
$$

By symmetry, we may assume that $t \leq s$.

Using (21), we deduce that

$$
2 s(1-t) \leq A(t, s) \leq 3 s(1-t) .
$$

To show (25), we separate the proof into three cases.

Case $1(r \leq t \leq s)$. In this case, one has

$$
\begin{aligned}
A(t, r)+A(r, s) & \geq 2(t+s)(1-r) \\
& \geq 2 s(1-t) \geq \frac{2}{3} A(t, s) .
\end{aligned}
$$

Case $2(t \leq r \leq s)$. We obtain

$$
\begin{aligned}
A(t, r)+A(r, s) & \geq 2 r(1-t)+2 s(1-r) \\
& \geq 2 s(1-t) \geq \frac{2}{3} A(t, s) .
\end{aligned}
$$


Case $3(t \leq s \leq r)$. One has

$$
\begin{aligned}
A(t, r)+A(r, s) & \geq 2 r(1-t)+2 r(1-s) \\
& \geq 2 s(1-t) \geq \frac{2}{3} A(t, s) .
\end{aligned}
$$

This completes the proof.

In the sequel, for any $q \in \mathscr{B}((0,1))$, we recall that

$$
\alpha_{q}:=\sup _{t, s \in(0,1)} \int_{0}^{1} \frac{G(t, r) G(r, s)}{G(t, s)}|q(r)| d r,
$$

and we denote by

$$
h_{1}(t)=t(1-t)^{2}, \quad h_{2}(t)=t^{2}(1-t), \quad \text { for } t \in[0,1] \text {. }
$$

Proposition 8. Let q be a function in $\mathscr{K}$; then

(i)

$$
\alpha_{q} \leq \frac{3}{2} \int_{0}^{1}(\rho(r))^{3}|q(r)| d r<\infty .
$$

(ii) For $t \in[0,1]$, one has

$$
\int_{0}^{1} G(t, s) h_{1}(s)|q(s)| d s \leq \alpha_{q} h_{1}(t) .
$$

(iii) For $t \in[0,1]$, one has

$$
\int_{0}^{1} G(t, s) h_{2}(s)|q(s)| d s \leq \alpha_{q} h_{2}(t) .
$$

In particular, for $t \in[0,1]$, one has

$$
\int_{0}^{1} G(t, s) \rho(s)|q(s)| d s \leq \alpha_{q} \rho(t) .
$$

Proof. Let $q$ be a function in $\mathscr{K}$.

(i) Using (24) and (23), one has, for $t, s \in(0,1)$,

$$
\begin{aligned}
\int_{0}^{1} \frac{G(t, r) G(r, s)}{G(t, s)}|q(r)| d r \leq & \frac{3}{2} \int_{0}^{1}\left(\frac{\rho(r)}{\rho(t)}\right)^{2} G(t, r) \\
& +\left(\frac{\rho(r)}{\rho(s)}\right)^{2} G(r, s)|q(r)| d r \\
\leq & \frac{3}{2} \int_{0}^{1}(\rho(r))^{3}|q(r)| d r
\end{aligned}
$$

Hence

$$
\alpha_{q} \leq \frac{3}{2} \int_{0}^{1}(\rho(r))^{3}|q(r)| d r<\infty .
$$

(ii) Since, for each $t, s \in(0,1)$, one has $\lim _{r \rightarrow 0}$ $(G(s, r) / G(t, r))=h_{1}(s) / h_{1}(t)$, then we deduce by Fatou's lemma and (12), that

$$
\begin{aligned}
& \int_{0}^{1} G(t, s) \frac{h_{1}(s)}{h_{1}(t)}|q(s)| d s \\
& \quad \leq \liminf _{r \rightarrow 0} \int_{0}^{1} G(t, s) \frac{G(s, r)}{G(t, r)}|q(s)| d s \leq \alpha_{q}
\end{aligned}
$$

which implies that, for $t \in[0,1]$,

$$
\int_{0}^{1} G(t, s) h_{1}(s)|q(s)| d s \leq \alpha_{q} h_{1}(t) .
$$

(iii) Similarly, we prove inequality (34) by observing that

$$
\lim _{r \rightarrow 1} \frac{G(s, r)}{G(t, r)}=\frac{h_{2}(s)}{h_{2}(t)} .
$$

Inequality (35) follows from Proposition 8 (ii)-(iii) and the fact that $\rho(t)=h_{1}(t)+h_{2}(t)$. This completes the proof.

\section{Proofs of Main Results}

In this section, we aim at proving Theorem 2 and Corollary 3. So, we need the following preliminaries results.

For a nonnegative function $q$ in $\mathscr{K}$ such that $\alpha_{q}<1$, we define the function $\mathscr{G}(t, s)$ on $[0,1] \times[0,1]$, by

$$
\mathscr{G}(t, s)=\sum_{n=0}^{\infty}(-1)^{n} G_{n}(t, s),
$$

where $G_{0}(t, s)=G(t, s)$ and

$$
G_{n}(t, s)=\int_{0}^{1} G(t, r) G_{n-1}(r, s) q(r) d r, \quad n \geq 1 .
$$

Next, we establish some inequalities on $G_{n}(t, s)$. In particular, we deduce that $\mathscr{G}(t, s)$ is well defined.

Lemma 9. Let $q$ be a nonnegative function in $\mathscr{K}$ such that $\alpha_{q}<$ 1 ; then, for each $n \geq 0$ and $t, s \in[0,1]$, one has

(i) $G_{n}(t, s) \leq \alpha_{q}^{n} G(t, s)$.

In particular, $\mathscr{G}(t, s)$ is well defined in $[0,1] \times[0,1]$.

(ii)

$$
L_{n}(\rho(t))^{2}(\rho(s))^{2} \leq G_{n}(t, s) \leq R_{n} \rho(t) \rho(s)(\rho(t) \wedge \rho(s)) ;
$$

where $L_{n}=\left(1 / 3^{n+1}\right)\left(\int_{0}^{1}(\rho(r))^{4} q(r) d r\right)^{n}$ and $R_{n}=$ $\left(1 / 2^{n+1}\right)\left(\int_{0}^{1}(\rho(r))^{3} q(r) d r\right)^{n}$.

(iii) $G_{n+1}(t, s)=\int_{0}^{1} G_{n}(t, r) G(r, s) q(r) d r$ and $G_{n}(t, s)=$ $G_{n}(s, t)$.

(iv) $\int_{0}^{1} \mathscr{G}(t, r) G(r, s) q(r) d r=\int_{0}^{1} G(t, r) \mathscr{G}(r, s) q(r) d r$. 
Proof. (i) The assertion is clear for $n=0$.

Assume that inequality in (i) holds for some $n \geq 0$; then by using (42) and (12), we obtain

$$
G_{n+1}(t, s) \leq \alpha_{q}^{n} \int_{0}^{1} G(t, r) G(r, s) q(r) d r \leq \alpha_{q}^{n+1} G(t, s) .
$$

Now, since $G_{n}(t, s) \leq \alpha_{q}^{n} G(t, s)$, it follows that $\mathscr{G}(t, s)$ is well defined in $[0,1] \times[0,1]$.

(ii) Using (22) and (42), we obtain (43) by induction.

(iii) Since $G(t, s)=G(s, t)$, then equalities in (iii) are clear for $n=0$. has

Assume that for a given integer $n \geq 1$ and $t, s \in[0,1]$, one

$$
\begin{gathered}
G_{n}(t, s)=\int_{0}^{1} G_{n-1}(t, r) G(r, s) q(r) d r \\
G_{n-1}(t, s)=G_{n-1}(s, t) .
\end{gathered}
$$

Using (42) and Fubini-Tonelli theorem, we obtain

$$
\begin{aligned}
& G_{n+1}(t, s) \\
& \quad=\int_{0}^{1} G(t, r)\left(\int_{0}^{1} G_{n-1}(r, \xi) G(\xi, s) q(\xi) d \xi\right) q(r) d r \\
& \quad=\int_{0}^{1}\left(\int_{0}^{1} G(t, r) G_{n-1}(r, \xi) q(r) d r\right) G(\xi, s) q(\xi) d \xi \\
& \quad=\int_{0}^{1} G_{n}(t, \xi) G(\xi, s) q(\xi) d \xi .
\end{aligned}
$$

On the other hand, by (42) and (45), we deduce that

$$
\begin{aligned}
G_{n}(t, s) & =\int_{0}^{1} G(t, r) G_{n-1}(r, s) q(r) d r \\
& =\int_{0}^{1} G_{n-1}(s, r) G(r, t) q(r) d r \\
& =G_{n}(s, t) .
\end{aligned}
$$

(iv) Let $n \geq 0$ and $t, r, s \in[0,1]$. By Lemma 9 (i) and (22), one has

$$
0 \leq G_{n}(t, r) G(r, s) q(r) \leq \alpha_{q}^{n} G(t, r) G(r, s) q(r) .
$$

Hence the series $\sum_{n \geq 0} \int_{0}^{1} G_{n}(t, r) G(r, s) q(r) d r$ converges.

So we deduce by the dominated convergence theorem and Lemma 9 (iii) that

$$
\begin{array}{rl}
\int_{0}^{1} & \mathscr{G}(t, r) G(r, s) q(r) d r \\
& =\sum_{n=0}^{\infty} \int_{0}^{1}(-1)^{n} G_{n}(t, r) G(r, s) q(r) d r \\
& =\sum_{n=0}^{\infty} \int_{0}^{1}(-1)^{n} G(t, r) G_{n}(r, s) q(r) d r \\
& =\int_{0}^{1} G(t, r) \mathscr{G}(r, s) q(r) d r .
\end{array}
$$

Proposition 10. Let $q$ be a nonnegative function in $\mathscr{K}$ such that $\alpha_{q}<1$. Then the function $(t, s) \rightarrow \mathscr{G}(t, s)$ is continuous on $[0,1] \times[0,1]$.

Proof. We claim that for $n \geq 0$, the function $G_{n}(t, s)$ is continuous on $[0,1] \times[0,1]$.

The assertion is clear for $n=0$.

Now assume that for a given integer $n \geq 1$, the function $(t, s) \rightarrow G_{n-1}(t, s)$ is continuous on $[0,1] \times[0,1]$. By $(42)$, one has

$$
G_{n}(t, s)=\int_{0}^{1} G(t, r) G_{n-1}(r, s) q(r) d r .
$$

Since the function $(t, s, r) \rightarrow G(t, r) G_{n-1}(r, s)$ is continuous on $[0,1] \times[0,1] \times[0,1]$ and by Lemma 9 (i) and (22)

$$
\begin{aligned}
G(t, r) G_{n-1}(r, s) q(r) & \leq \alpha_{q}^{n-1} G(t, r) G(r, s) q(r) \\
& \leq(\rho(r))^{3} q(r)
\end{aligned}
$$

we deduce by the dominated convergence theorem that $(t, s) \rightarrow G_{n}(t, s)$ is continuous on $[0,1] \times[0,1]$.

This proves our claim.

Now by Lemma 9(i) and (22), one has, for each $t, s \in$ $[0,1]$,

$$
G_{n}(t, s) \leq \alpha_{q}^{n} G(t, s) \leq \frac{1}{2} \rho(t)(\rho(s))^{2} \alpha_{q}^{n} \leq \alpha_{q}^{n} .
$$

This implies that the series $\sum_{n \geq 0}(-1)^{n} G_{n}(t, s)$ is uniformly convergent on $[0,1] \times[0,1]$ and therefore the function $(t, s) \rightarrow \mathscr{G}(t, s)$ is continuous on $[0,1] \times[0,1]$.

The proof is completed.

Lemma 11. Let $q$ be a nonnegative function in $\mathscr{K}$ such that $\alpha_{q} \leq 1 / 2$. Then for $t, s$ in $[0,1]$, one has

$$
\left(1-\alpha_{q}\right) G(t, s) \leq \mathscr{G}(t, s) \leq G(t, s) .
$$

Proof. Since $\alpha_{q} \leq 1 / 2$, we deduce from Lemma 9 (i) that

$$
|\mathscr{G}(t, s)| \leq \sum_{n=0}^{\infty}\left(\alpha_{q}\right)^{n} G(t, s)=\frac{1}{1-\alpha_{q}} G(t, s) .
$$

On the other hand, from the expression of $\mathscr{G}$, one has

$$
\mathscr{G}(t, s)=G(t, s)-\sum_{n=0}^{\infty}(-1)^{n} G_{n+1}(t, s) .
$$

Since the series $\sum_{n \geq 0} \int_{0}^{1} G(t, r) G_{n}(r, s) q(r) d r$ is convergent, we deduce by (55) and (42) that

$$
\begin{aligned}
\mathscr{G}(t, s) & =G(t, s)-\sum_{n=0}^{\infty}(-1)^{n} \int_{0}^{1} G(t, r) G_{n}(r, s) q(r) d r \\
& =G(t, s)-\int_{0}^{1} G(t, r)\left(\sum_{n=0}^{\infty}(-1)^{n} G_{n}(r, s)\right) q(r) d r .
\end{aligned}
$$


That is,

$$
\mathscr{G}(t, s)=G(t, s)-V(q \mathscr{G}(\cdot, s))(t) .
$$

Now from (54) and Lemma 9(i) (with $n=1$ ), we obtain

$$
\begin{aligned}
V(q \mathscr{G}(\cdot, s))(t) & \leq \frac{1}{1-\alpha_{q}} V(q G(\cdot, s))(t) \\
& =\frac{1}{1-\alpha_{q}} G_{1}(t, s) \leq \frac{\alpha_{q}}{1-\alpha_{q}} G(t, s) .
\end{aligned}
$$

This implies that

$$
\mathscr{G}(t, s) \geq G(t, s)-\frac{\alpha_{q}}{1-\alpha_{q}} G(t, s)=\frac{1-2 \alpha_{q}}{1-\alpha_{q}} G(t, s) \geq 0 .
$$

So it follows that $\mathscr{G}(t, s) \leq G(t, s)$ and by (57) and Lemma 9(i) (with $n=1$ ), one has

$$
\mathscr{G}(t, s) \geq G(t, s)-V(q G(\cdot, s))(t) \geq\left(1-\alpha_{q}\right) G(t, s)
$$

In the sequel, for a given nonnegative function $q \in \mathscr{K}$ such that $\alpha_{q} \leq 1 / 2$, we define the operator $V_{q}: \mathscr{B}^{+}((0,1)) \rightarrow$ $\mathscr{B}^{+}((0,1))$ by

$$
V_{q} \psi(t)=\int_{0}^{1} \mathscr{G}(t, s) \psi(s) d s, \quad t \in[0,1] .
$$

Using (53) and (22), we obtain the following.

Corollary 12. Let $q$ be a nonnegative function in $\mathscr{K}$ such that $\alpha_{q} \leq 1 / 2$ and $\psi \in \mathscr{B}^{+}((0,1))$; then the following statements are equivalent.

(i) The function $t \rightarrow V_{q} \psi(t)$ is continuous on $[0,1]$.

(ii) The integral $\int_{0}^{1}(\rho(s))^{2} \psi(s) d s$ converges.

Next, we will prove that the kernel $V_{q}$ satisfies the following resolvent equation.

Lemma 13. Let $q$ be a nonnegative function in $\mathscr{K}$ such that $\alpha_{q} \leq 1 / 2$ and $\psi \in \mathscr{B}^{+}((0,1))$. Then $V_{q} \psi$ satisfies the following resolvent equation:

$$
V \psi=V_{q} \psi+V_{q}(q V \psi)=V_{q} \psi+V\left(q V_{q} \psi\right) .
$$

In particular, if $V(q \psi)<\infty$, one has

$$
\begin{aligned}
(I & \left.-V_{q}(q \cdot)\right)(I+V(q \cdot)) \psi \\
& =(I+V(q \cdot))\left(I-V_{q}(q \cdot)\right) \psi=\psi .
\end{aligned}
$$

Proof. Let $t, s \in[0,1]$, then by (57) one has

$$
G(t, s)=\mathscr{G}(t, s)+V(q \mathscr{G}(\cdot, s))(t),
$$

which implies by Fubini-Tonelli theorem that, for $\psi \in$ $\mathscr{B}^{+}((0,1))$,

$$
\begin{aligned}
V \psi(t) & =\int_{0}^{1}(\mathscr{G}(t, s)+V(q \mathscr{G}(\cdot, s))(t)) \psi(s) d s \\
& =V_{q} \psi(t)+V\left(q V_{q} \psi\right)(t) .
\end{aligned}
$$

On the other hand, by Lemma 9 (iii) and Fubini-Tonelli theorem, we obtain for $\psi \in \mathscr{B}^{+}((0,1))$ and $t \in[0,1]$

$$
\begin{aligned}
\int_{0}^{1} \int_{0}^{1} \mathscr{G}(t, r) G(r, s) \psi(s) q(r) d r d s \\
\quad=\int_{0}^{1} \int_{0}^{1} G(t, r) \mathscr{G}(r, s) \psi(s) q(r) d r d s
\end{aligned}
$$

that is,

$$
V_{q}(q V \psi)(t)=V\left(q V_{q} \psi\right)(t)
$$

So we obtain

$$
V \psi=V_{q} \psi+V\left(q V_{q} \psi\right)=V_{q} \psi+V_{q}(q V \psi)(t) .
$$

This completes the proof.

Proposition 14. Let $q$ be a nonnegative function in $\mathscr{K} \cap \mathscr{C}(0,1)$ such that $\alpha_{q} \leq 1 / 2$ and let $\psi \in \mathscr{B}^{+}((0,1))$ such that $t \rightarrow$ $(\rho(t))^{2} \psi(t)$ is continuous and integrable on $(0,1)$. Then $V_{q} \psi$ is the unique nonnegative solution in $C^{4}((0,1)) \cap C([0,1])$ of the perturbed fourth-order equation (13) satisfying

$$
\left(1-\alpha_{q}\right) V \psi \leq V_{q} \psi \leq V \psi \text {. }
$$

Proof. It is clear by Corollary 12 that the function $t \rightarrow$ $q(t) V_{q} \psi(t)$ is continuous on $(0,1)$. Using (62) and (22), there exists a nonnegative constant $c$ such that

$$
V_{q} \psi(t) \leq V \psi(t) \leq \frac{1}{2} \int_{0}^{1} \rho(t)(\rho(s))^{2} \psi(s) d s \leq c \rho(t) .
$$

So we deduce that

$$
\int_{0}^{1}(\rho(s))^{2} q(s) V_{q} \psi(s) d s \leq c \int_{0}^{1}(\rho(s))^{3} q(s) d s<\infty .
$$

Hence by using Remark 4 , the function $u=V_{q} \psi=V \psi-$ $V\left(q V_{q} \psi\right)$ satisfies the equation

$$
\begin{gathered}
u^{(4)}(t)=\psi(t)-q(t) u(t), \quad t \in(0,1), \\
u(0)=u(1)=u^{\prime}(0)=u^{\prime}(1)=0,
\end{gathered}
$$

and by integration inequalities (53), we obtain (69).

It remains to prove the uniqueness. Assume that $v$ is another nonnegative solution in $C^{4}((0,1)) \cap C([0,1])$ of problem (13) satisfying (69).

Since the function $t \rightarrow q(t) v(t)$ is continuous on $(0,1)$ and by (69) and (70), the function $t \rightarrow(\rho(t))^{2} q(t) v(t)$ is 
integrable on $(0,1)$; then it follows by Remark 4 that the function $\widetilde{v}:=v+V(q v)$ satisfies

$$
\begin{gathered}
\widetilde{v}^{(4)}(t)=\psi(t), \quad t \in(0,1), \\
\widetilde{v}(0)=\widetilde{v}(1)=\widetilde{v}^{\prime}(0)=\widetilde{v}^{\prime}(1)=0 .
\end{gathered}
$$

From the uniqueness in Remark 4, we deduce that

$$
\widetilde{v}:=v+V(q v)=V \psi .
$$

Hence

$$
(I+V(q \cdot))(v-u)=0 .
$$

Now since by (69), (70), and (35), one has

$$
V(q|v-u|) \leq 2 c V(q \rho) \leq 2 c \alpha_{q} \rho<\infty,
$$

then by (63), we deduce that $u=v$. This completes the proof.

Proof of Theorem 2. Let $a \geq 0$ and $b \geq 0$ with $a+b>0$ and recall that

$$
\omega(t)=a t(1-t)^{2}+b t^{2}(1-t)=a h_{1}(t)+b h_{2}(t) .
$$

Since $\varphi$ satisfies $\left(\mathrm{H}_{2}\right)$, then there exists a positive function $q$ in $\mathscr{K} \cap C(0,1)$ such that $\alpha_{q} \leq 1 / 2$ and for each $t \in(0,1)$, the map $s \rightarrow s(q(t)-\varphi(t, s \omega(t)))$ is nondecreasing on $[0,1]$.

Let

$$
\Lambda:=\left\{u \in \mathscr{B}^{+}((0,1)):\left(1-\alpha_{q}\right) \omega \leq u \leq \omega\right\},
$$

and define the operator $T$ on $\Lambda$ by

$$
T u=\omega-V_{q}(q \omega)+V_{q}((q-\varphi(\cdot, u)) u) .
$$

By (62) and Proposition 8, one has

$$
V_{q}(q \omega) \leq V(q \omega) \leq \alpha_{q} \omega \leq \omega,
$$

and by $\left(H_{2}\right)$, we obtain

$$
0 \leq \varphi(\cdot, u) \leq q, \quad \forall u \in \Lambda
$$

So we claim that $\Lambda$ is invariant under $T$. Indeed, using (81) and (80), one has for $u \in \Lambda$

$$
\begin{gathered}
T u \leq \omega-V_{q}(q \omega)+V_{q}(q u) \leq \omega, \\
T u \geq \omega-V_{q}(q \omega) \\
\geq\left(1-\alpha_{q}\right) \omega .
\end{gathered}
$$

Next, we will prove that the operator $T$ is nondecreasing on $\Lambda$. Indeed, let $u, v \in \Lambda$ be such that $u \leq v$. Since the map $s \rightarrow s(q(t)-\varphi(t, s \omega(t)))$ is nondecreasing on $[0,1]$, for $t \in$ $(0,1)$, we obtain

$$
T v-T u=V_{q}([v(q-\varphi(\cdot, v))-u(q-\varphi(\cdot, u))]) \geq 0 .
$$

Now, we consider the sequence $\left(u_{n}\right)$ defined by $u_{0}=(1-$ $\left.\alpha_{q}\right) \omega$ and $u_{n+1}=T u_{n}$, for $n \in \mathbb{N}$. Since $\Lambda$ is invariant under $T$, one has $u_{1}=T u_{0} \geq u_{0}$ and by the monotonicity of $T$, we deduce that

$$
\left(1-\alpha_{q}\right) \omega=u_{0} \leq u_{1} \leq \cdots \leq u_{n} \leq u_{n+1} \leq \omega .
$$

Hence by dominated convergence theorem and hypotheses $\left(H_{1}\right)-\left(H_{2}\right)$, we conclude that the sequence $\left(u_{n}\right)$ converges to a function $u \in \Lambda$ satisfying

$$
u=\left(I-V_{q}(q \cdot)\right) \omega+V_{q}((q-\varphi(\cdot, u)) u) .
$$

That is,

$$
\left(I-V_{q}(q \cdot)\right) u=\left(I-V_{q}(q \cdot)\right) \omega-V_{q}(u \varphi(\cdot, u)) .
$$

On the other hand, since by (80), one has $V(q u) \leq$ $V(q \omega) \leq \omega<\infty$, then by applying the operator $(I+V(q \cdot))$ on both sides of the above equality and using (62) and (63), we conclude that $u$ satisfies

$$
u=\omega-V(u \varphi(\cdot, u))
$$

Next we aim at proving that $u$ is a solution of problem (5). To this end, we remark by (81) and (9) that

$$
u \varphi(\cdot, u) \leq q \omega \leq \max (a, b) q \rho .
$$

This implies by Corollary 6 (ii) that the function $t \rightarrow$ $V(u \varphi(., u))(t)$ is continuous on $[0,1]$ and so by $(87), u$ is continuous on $[0,1]$.

Now, since by $\left(H_{1}\right)$ and (88), the function $t \rightarrow$ $(\rho(t))^{2} u(t) \varphi(t, u(t))$ is continuous and integrable on $(0,1)$, we conclude by Remark 4 that $u$ is the required solution.

It remains to prove that under condition $\left(\mathrm{H}_{3}\right), u$ is the unique solution to problem (5) satisfying (14). Assume that $v$ is another nonnegative solution in $C^{4}((0,1)) \cap C([0,1])$ to problem (5) satisfying (14). Since $v \leq \omega$, we deduce by (88) that

$$
0 \leq v \varphi(\cdot, v) \leq q \omega \leq \max (a, b) q \rho .
$$

So the function $t \rightarrow(\rho(t))^{2} v(t) \varphi(t, v(t))$ is continuous and integrable on $(0,1)$ and by Remark 4 , we conclude that the function $\widetilde{v}:=v+V(v \varphi(., v))$ satisfies

$$
\begin{gathered}
\widetilde{v}^{(4)}(t)=0, \quad t \in(0,1), \\
\widetilde{v}(0)=\widetilde{v}(1)=0, \quad \widetilde{v}^{\prime}(0)=a, \quad \widetilde{v}^{\prime}(1)=-b .
\end{gathered}
$$

From the uniqueness in problem (8), we deduce that

$$
v=\omega-V(v \varphi(\cdot, v)) .
$$

Now let $h$ be the function defined on $(0,1)$ by

$$
h(t)= \begin{cases}\frac{v(t) \varphi(t, v(t))-u(t) \varphi(t, u(t))}{v(t)-u(t)} & \text { if } v(t) \neq u(t), \\ 0 & \text { if } v(t)=u(t) .\end{cases}
$$



has

Then by $\left(H_{3}\right), h \in \mathscr{B}^{+}((0,1))$ and by $(87)$ and $(91)$, one

$$
(I+V(h \cdot))(v-u)=0 .
$$

On the other hand, by $\left(\mathrm{H}_{2}\right)$, we remark that $h \leq q$ and by (80) we deduce that

$$
V(h|v-u|) \leq 2 V(q \omega) \leq 2 \alpha_{q} \omega<\infty .
$$
proof.

Hence by (63), we conclude that $u=v$. This completes the Proof of Corollary 3. Let $\varphi(t, s)=\lambda p(t) f(s), \theta(s)=s f(s)$, and $\widetilde{p}(t)=p(t) \max _{0 \leq \xi \leq \omega(t)} \theta^{\prime}(\xi)$. It is clear that hypotheses $\left(H_{1}\right)$ and $\left(H_{3}\right)$ are satisfied. Since the function $q(t):=\lambda \widetilde{p}(t)$ belongs to the class $\mathscr{R}$, one has $\alpha_{q} \leq 1 / 2$ for $\lambda \in\left[0,1 / 2 \alpha_{\tilde{p}}\right)$. Moreover, by a simple computation, for $s \in[0,1]$ and $t \in$ $(0,1)$, we obtain

$$
\frac{d}{d s}[s(q(t)-\varphi(t, s \omega(t)))]=q(t)-\lambda p(t) \theta^{\prime}(s \omega(t)) \geq 0 .
$$

This implies that the function $\varphi$ satisfies hypothesis $\left(\mathrm{H}_{2}\right)$. So the result follows by Theorem 2 .

Example 15. Let $a \geq 0$ and $b \geq 0$ with $a+b>0$. Let $\sigma \geq 0$, and $p$ be a positive continuous function on $(0,1)$ such that

$$
\int_{0}^{1} r^{3+\sigma}(1-r)^{3+\sigma} p(r) d r<\infty .
$$

Since the function $\widetilde{p}(t):=(\sigma+1) p(t)(\omega(t))^{\sigma}$ belongs to the class $\mathscr{K}$, then, for $\lambda \in\left[0,1 / 2 \alpha_{\tilde{p}}\right)$, the problem

$$
\begin{aligned}
& u^{(4)}(t)+\lambda p(t) u^{\sigma+1}(t)=0, \quad t \in(0,1), \\
& u(0)=u(1)=0, \quad u^{\prime}(0)=a, \quad u^{\prime}(1)=-b
\end{aligned}
$$

has a unique positive solution $u$ in $C^{4}((0,1)) \cap C([0,1])$ satisfying

$$
\left(1-\lambda \alpha_{\tilde{p}}\right) \omega(t) \leq u(t) \leq \omega(t), \quad t \in[0,1] .
$$

Example 16. Let $a \geq 0$ and $b \geq 0$ with $a+b>0$. Let $\sigma \geq 0, \gamma>$ 0 , and $p$ be a positive continuous function on $(0,1)$ such that

$$
\int_{0}^{1} r^{3+\sigma+\gamma}(1-r)^{3+\sigma+\gamma} p(r) d r<\infty
$$

Let $\theta(s)=s^{\sigma+1} \log \left(1+s^{\gamma}\right)$. Since the function $\widetilde{p}(t):=$ $p(t) \max _{0 \leq \xi \leq \omega(t)} \theta^{\prime}(\xi) \in \mathscr{K}$, then, for $\lambda \in\left[0,1 / 2 \alpha_{\tilde{p}}\right)$, the problem

$$
\begin{gathered}
u^{(4)}(t)+\lambda p(t) u^{\sigma+1}(t) \log \left(1+u^{\gamma}(t)\right)=0, \quad t \in(0,1), \\
u(0)=u(1)=0, \quad u^{\prime}(0)=a, \quad u^{\prime}(1)=-b
\end{gathered}
$$

has a unique positive solution $u$ in $C^{4}((0,1)) \cap C([0,1])$ satisfying

$$
\left(1-\lambda \alpha_{\tilde{p}}\right) \omega(t) \leq u(t) \leq \omega(t), \quad t \in[0,1] .
$$

\section{Conflict of Interests}

The authors declare that there is no conflict of interests regarding the publication of this paper.

\section{Acknowledgments}

The authors thank the referees for their careful reading of the paper. This project was supported by King Saud University, Deanship of Scientific Research, College of Science Research Center.

\section{References}

[1] R. Ma and C. C. Tisdell, "Positive solutions of singular sublinear fourth-order boundary value problems," Applicable Analysis, vol. 84, no. 12, pp. 1199-1221, 2005.

[2] R. P. Agarwal and Y. M. Chow, "Iterative methods for a fourth order boundary value problem," Journal of Computational and Applied Mathematics, vol. 10, no. 2, pp. 203-217, 1984.

[3] R. P. Agarwal, D. O'Regan, and P. J. Y. Wong, Positive Solutions of Differential, Difference and Integral Equations, Kluwer Academic, Boston, Mass, USA, 1999.

[4] R. S. Alsaedi, "Existence and global behavior of positive solutions for some fourth order boundary value problems," Abstract and Applied Analysis, vol. 2014, Article ID 657926, 5 pages, 2014.

[5] Z. Bai and H. Wang, "On positive solutions of some nonlinear fourth-order beam equations," Journal of Mathematical Analysis and Applications, vol. 270, no. 2, pp. 357-368, 2002.

[6] A. Cabada and R. R. Enguiça, "Positive solutions of fourth order problems with clamped beam boundary conditions," Nonlinear Analysis: Theory, Methods \& Applications, vol. 74, no. 10, pp. 3112-3122, 2011.

[7] Y. J. Cui, J. X. Sun, and Y. M. Zou, "Positive solutions of singular boundary value problems of fourth-order differential equations," Journal of Mathematical Research and Exposition, vol. 29, no. 2, pp. 376-380, 2009.

[8] Y. Cui and Y. Zou, "Positive solutions of singular fourth-order boundary-value problems," Electronic Journal of Differential Equations, vol. 39, pp. 1-10, 2006.

[9] R. Dalmasso, "Uniqueness of positive solutions for some nonlinear fourth-order equations," Journal of Mathematical Analysis and Applications, vol. 201, no. 1, pp. 152-168, 1996.

[10] J. M. do Ó, S. Lorca, and P. Ubilla, "Multiplicity of solutions for a class of non-homogeneous fourth-order boundary value problems," Applied Mathematics Letters, vol. 21, no. 3, pp. 279286, 2008.

[11] J. R. Graef, L. Kong, and B. Yang, "Existence, nonexistence, and uniqueness of positive solutions to a three point fourth order boundary value problem," Nonlinear Studies, vol. 18, no. 4, pp. 565-575, 2011.

[12] L. Kong and J. S. W. Wong, "Positive solutions for higher order multi-point boundary value problems with nonhomogeneous boundary conditions," Journal of Mathematical Analysis and Applications, vol. 367, no. 2, pp. 588-611, 2010.

[13] P. Korman, "Uniqueness and exact multiplicity of solutions for a class of fourth-order semilinear problems," Proceedings of the Royal Society of Edinburgh A: Mathematics, vol. 134, no. 1, pp. 179-190, 2004. 
[14] R. Y. Ma and H. P. Wu, "Positive solutions of a fourth-order twopoint boundary value problem," Acta Mathematica Scientia, vol. 22, no. 2, pp. 244-249, 2002.

[15] Q. Yao, "Positive solutions for eigenvalue problems of fourthorder elastic beam equations," Applied Mathematics Letters. An International Journal of Rapid Publication, vol. 17, no. 2, pp. 237243, 2004. 


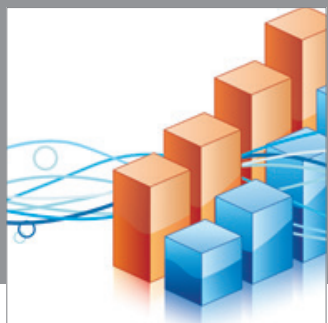

Advances in

Operations Research

mansans

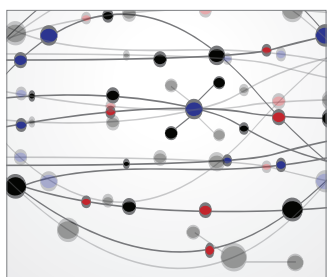

The Scientific World Journal
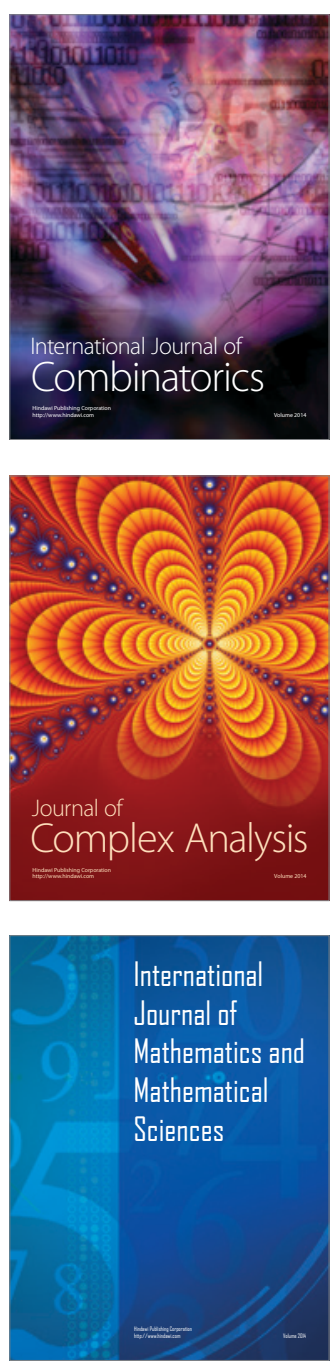
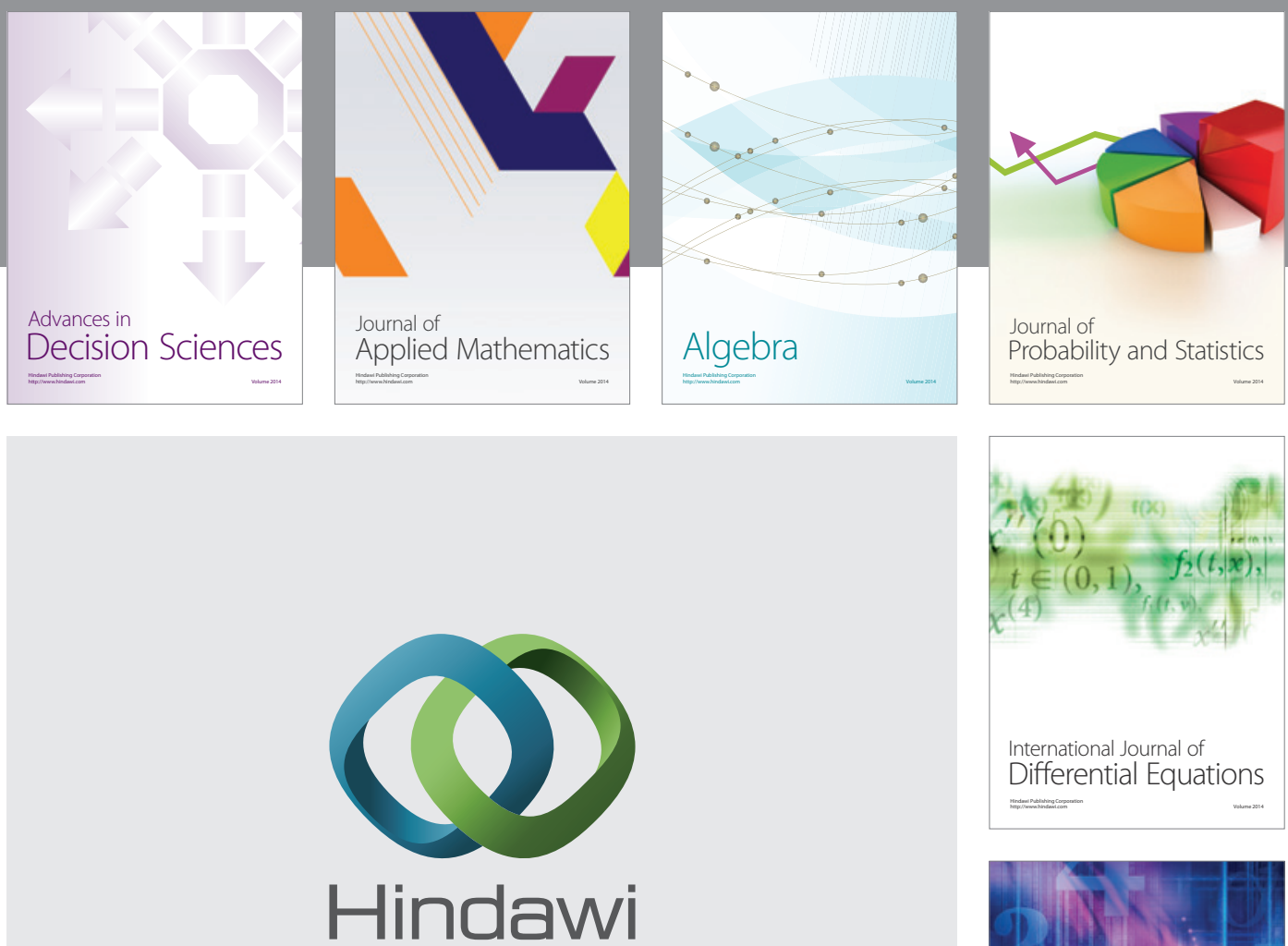

Submit your manuscripts at http://www.hindawi.com
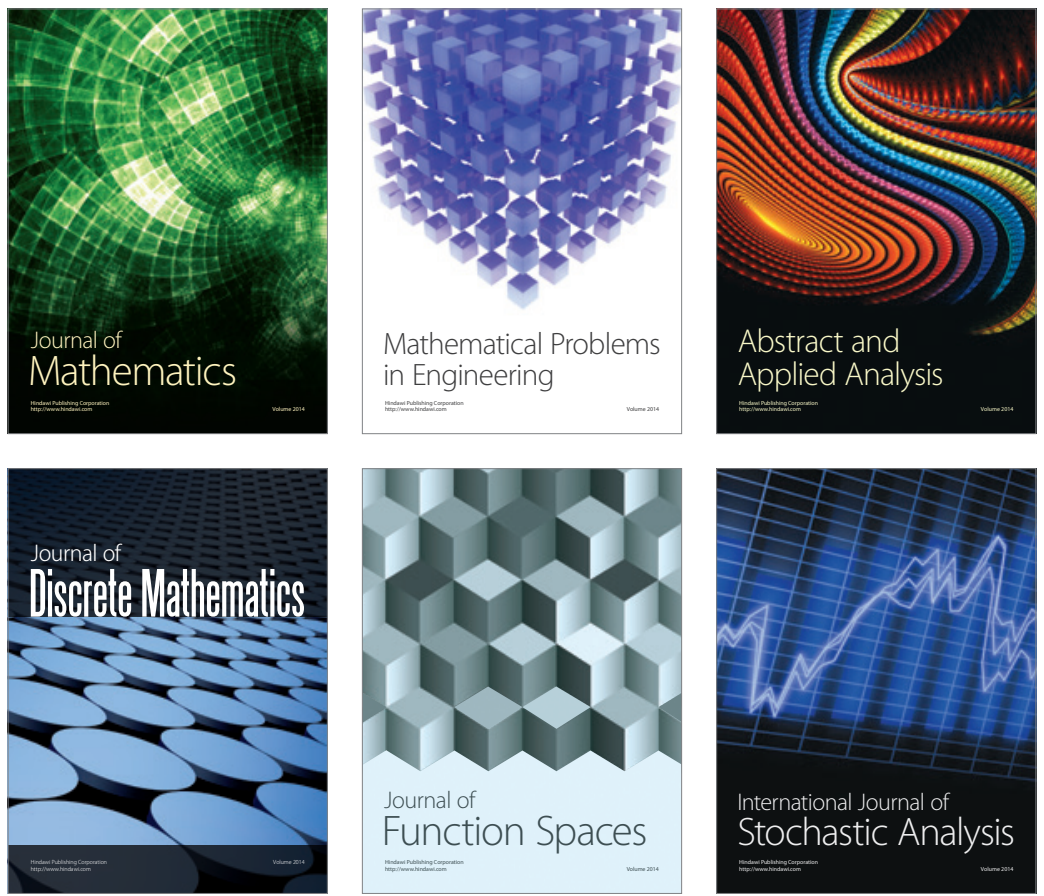

Journal of

Function Spaces

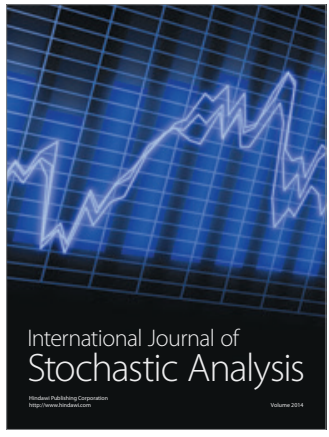

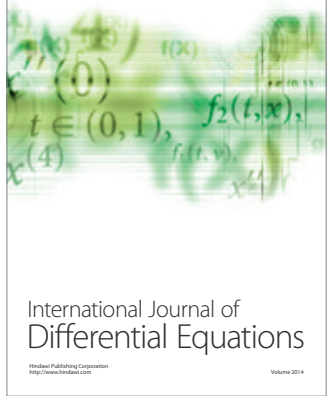
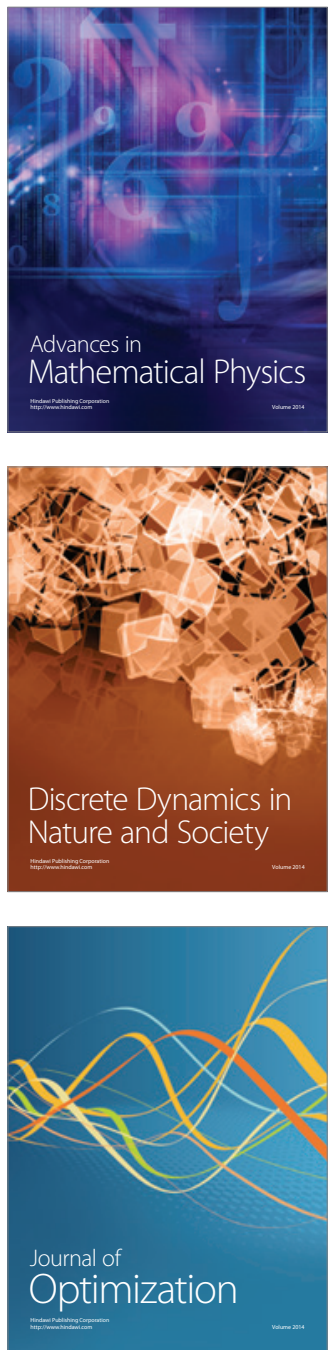